\title{
Comparative Evaluation of Open Source Urban Simulation Models Applied to Colombo City and Environs in Sri Lanka
}

\author{
Jayasinghe, P., ${ }^{1}$ Kantakumar, L. N., ${ }^{2}$ Raghavan, V. ${ }^{3}$ and Yonezawa, G. ${ }^{3}$ \\ ${ }^{1}$ Graduate School for Creative Cities, Osaka City University, 3-3-138 Sugimoto, Sumiyoshi-ku, Osaka 558- \\ 8585, Japan, E-mail: jayasinghe.pavithrap@gmail.com \\ ${ }^{2}$ Bharati Vidyapeeth Deemed University, Institute of Environment Education and Research, Pune 411043, \\ India, E-mail: Lakshmikanth@bvieer.edu.in \\ ${ }^{3}$ Graduate School of Engineering, Osaka City University, 3-3-138 Sugimoto, Sumiyoshi-ku, Osaka 558-8585, \\ Japan \\ DOI: https://doi.org/10.52939/ijg.v17i3.1897
}

\begin{abstract}
Availability of a variety of urban growth models make model selection to be an important factor in urban simulation studies. In this regard, a comparative evaluation of available urban growth models helps to choose a suitable model for the study area. Thus, we selected three open-source simulation models namely FUTURES, SLEUTH and MOLUSCE to compare in their simplest state to provide a guidance for selection of an urban growth model for Colombo. The urban extent maps of 1997, 2005, 2008, 2014 and 2019 derived from Landsat imageries were used in calibration and validation of models. Models were implemented with the minimum required data with default settings. The simulation results indicate that the estimated quantity of urban growth $\left(148.91 \mathrm{~km}^{2}\right)$ during 2008-2019 by FUTURES model is matching closely with observed urban growth (127.37 $\left.\mathrm{km}^{2}\right)$ during 2008-2019. On the other hand, the SLEUTH model showed an overestimation (250.56 $\left.\mathrm{km}^{2}\right)$ and MOLUSCE showed an underestimation $\left(77.11 \mathrm{~km}^{2}\right)$. Further, the spatial accuracy of urban growth simulation of SLEUTH (Figure of Merit $=0.26$ ) is relatively better in comparison to FUTURES (0.20) and MOLUSCE (0.20). Considering the tradeoff between computational overheads and obtained results, FUTURES could be a good choice over SLEUTH and MOLUSCE, when these models implemented in their simplest form with minimum required datasets. As a future work, we propose the incorporation of exclusion factor for potential surface generation to mitigate the overestimation of urban areas in SLUETH.
\end{abstract}

\section{Introduction}

The land use change driven by urban growth is one of the most influential transformation that can affect the natural and social cohesion (Sandamali et al., 2018). Urban growth models are useful tools for simplifying complex socioeconomic and biophysical forces that influence the rate and spatial pattern of land use change driven by the urbanization. These models are helpful in identifying the driving forces of urban growth dynamics and capable of simulating the anticipating future urbanization based on population growth projections, economic trends, and governance systems (Gounaridis et al., 2018, and Meentemeyer et al., 2013). Numerous urban growth simulation models with diverse level of sophistication have been developed and their efficacy in accurately predicting urban growth have been demonstrated (Clarke et al., 1997, Jin and Lee, 2018, Meentemeyer et al., 2013 and Van Berkel et al., 2019). Selecting an appropriate model that serves the purpose of the study is often arbitrary or depends upon the availability of inputs required to run the model or on the arbitrary choice made by the researchers. Several studies offer guidelines for prior understanding on performance of model using validation metrics related to quantity and allocation (Olofsson et al., 2014, Pontius and Malanson, 2005 and Pontius and Millones, 2011). However, limited number of studies comparing model simulation efficacies and trade-offs leads to a critical research gap (Pickard et al., 2017).

Comparative evaluation of models provides a guidance in selection of urban growth model and highlight trade-offs between selecting one model over another. Therefore, this study aims to evaluate the simulation outputs from three Open Source urban growth models namely, FUTURES (FUTure UrbanRegional Environment Simulation), MOLUSCE (Modules for Land Use Change Simulations) and SLEUTH (Slope, Land use, Exclusion, Urban, Transport and Hillshade) using Colombo as study area. Presently, FUTURES has been tested and 
validated for urban areas in the United States to evaluate land development dynamics in the rapidly expanding metropolitan region of Charlotte, North Carolina (Meentemeyer et al., 2013) and to analyze the impacts of urbanization on natural resources under different conservation strategies (Dorning et al., 2015). Van Berkel et al., (2019) applied FUTURES to evaluate urban growth strategies that encourage settlement densification in the South Atlantic States to mitigate increasing urban sprawl. Further, it was adopted to simulate different urban growth strategies in the South Atlantic States in USA and propose alternatives to mitigate the region's increasing sprawl (Shoemaker et al., 2019). First application of FUTURES, in the context of Asian cities was carried out in Colombo city Sri Lanka (Jayasinghe et al., 2021) and the results were evaluated to provide recommendations for development plans of Colombo city.

The selection of SLEUTH is due to its extensive application to study the future urban growth of cities around the world (Chaudhuri and Clarke, 2013 and Ilyassova et al., 2019). Several studies were used SLEUTH for impact assessment of various urban scenarios including South Asia (Chaudhuri and Clarke, 2019, Kantakumar et al., 2011, Sandamali et al., 2018 and Saxena and Jat, 2020). The selection of MOLUSCE is due to the reason of its availability as a plugin in the widely used QGIS software. Nugroho et al., (2018) applied the MOLUSCE model to predict land cover changes and direction of spatial distribution in Malang City, Indonesia. Hakim et al., (2019) used MOLUSCE to identify the spatial dynamics and predict LULC changes in Makassar city, Indonesia. In comparison to FUTURES and SLEUTH, MOLUSCE is not as widely tested. MOLUSCE has a user-friendly graphical user interface (GUI) interface and easy to implement. We considered these three models to evaluate their efficacy to simulate the urban growth of Colombo. We also focus on comparing the results of the models using minimum datasets and in their simplest state.

\section{Material and Methods}

\subsection{Study Area}

Colombo city core area (Colombo port and surrounding) and its adjacent suburbs were selected as the study area considering rapid urbanization during past few decades. Colombo is the highly urbanized area as well as located in close proximity to the capital of Sri Lanka. It depicts highest density of population and infrastructure network in Sri Lanka. Urban areas are densely concentrated within $35 \mathrm{~km}$ buffer zone from Colombo central business district (CBD) covering 35 Divisional Secretariat Divisions (DSD). Thus, we selected the rectangular extent encompassing these $35 \mathrm{DSD}$ as the study area (Figure 1).

\subsection{Input Data}

As major data input, urban area maps of 1997, 2005, 2008, 2014 and 2019 were derived from freely available Landsat sensor series $30 \mathrm{~m}$ data (Table 1). GRASS GIS r.learn.ml module with k-nearest neighbors algorithm (Brenning, 2012) was used to produce Land Cover (LC) classification maps using from Landsat Thematic Mapper (TM) and Operational Land Imager (OLI) data. A minimum of 50 training samples for each class were digitized and used in the classification. Initially classified LC maps were cross checked through careful and rigorous visual inspection, comparing them with the original Landsat and Google Earth imageries. The training samples were further refined to extract the final LC maps. LC maps for 1997, 2005, 2008, 2014 and 2019 were re-classified to derive urban and non-urban categories considering built-up density.

Table 1: Input data

\begin{tabular}{|l|l|l|}
\hline \multicolumn{1}{|c|}{ Data } & \multicolumn{1}{c|}{ Year } & \multicolumn{1}{c|}{ Data source } \\
\hline Landsat 5 TM & $1997,2005,2008$ & EarthExplorer, USGS \\
\hline Landsat 8 OLI & 2019 & EarthExplorer, USGS \\
\hline Population & $1991,2001,2012$ & Dept. of Census \& Statistics \\
\hline Road network & 2013 & JICA \\
\hline Water bodies & 2013 & JICA \\
\hline SRTM 30m DEM & 2000 & EarthExplorer, USGS \\
\hline $\begin{array}{l}\text { Social infrastructure } \\
\text { (Hospitals, schools) }\end{array}$ & 2004 & Survey Dept. \\
\hline Growth centers & 2010 & Survey Dept. \\
\hline Administrative boundary & 2010 & Survey Dept. \\
\hline
\end{tabular}




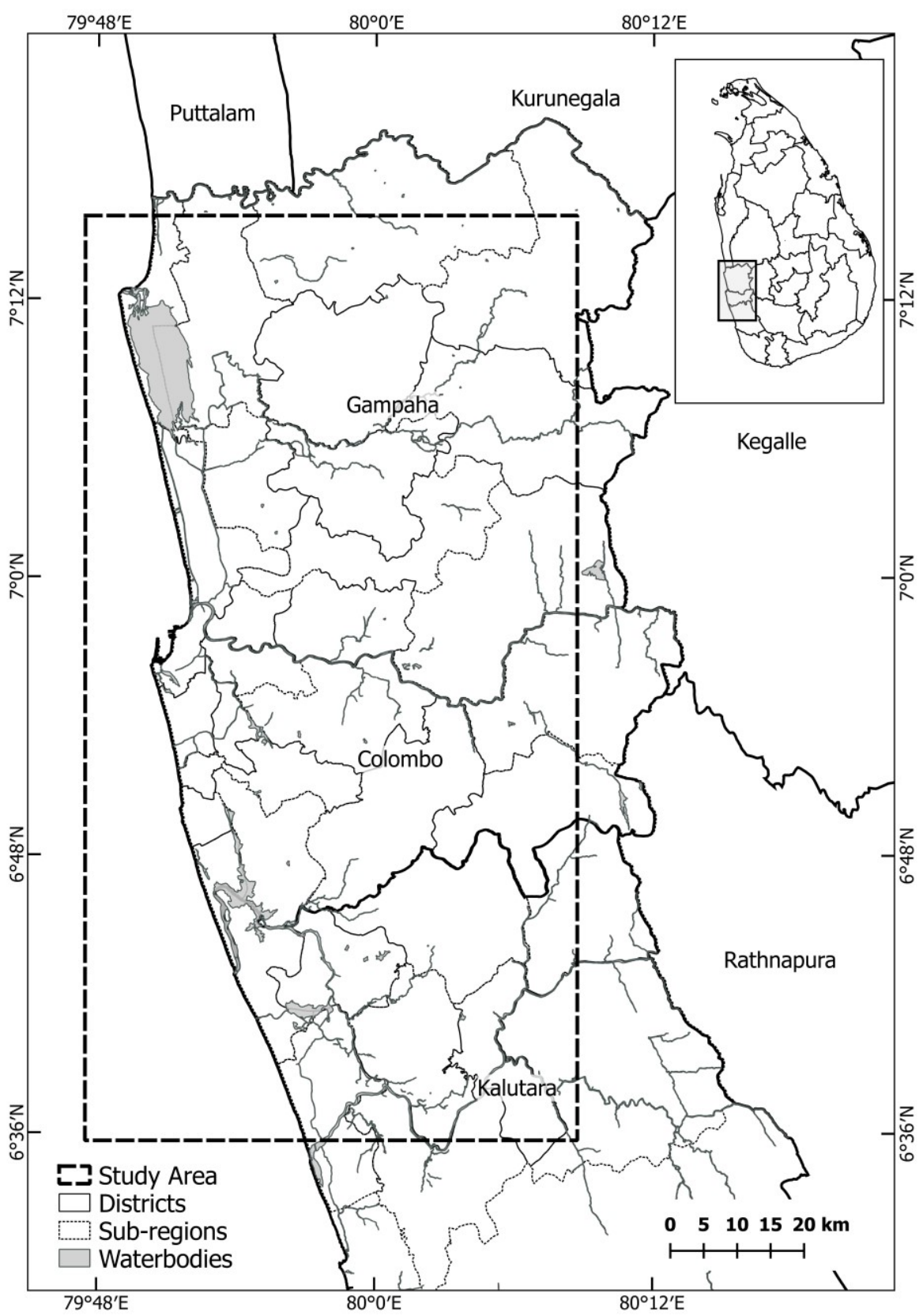

Figure 1: Location of the study area

Proportion of built-up pixels within $1 \mathrm{~km}^{2}$ area was calculated and used to produce three categories namely urban ( $\geq 50 \%$ built-up), sub-urban $(<50 \%$ and $\geq 10 \%$ built-up) and rural ( $<10 \%$ built-up). Further, considering spatial distribution and ground understanding, dense urban and sub-urban categories were combined into a single class labeled as 'Urban' and remaining areas were aggregated and labelled as 'Non-Urban'. Subsequently, urban class (Figure 2) was used for urban growth modeling. The FUTURES and MOLUSE models use predictor variables to estimate the transition potential. Thus, distance to roads, growth centers, hospitals, schools, environmental sensitive areas and percentage slope were used in this study as predictor variables (Figure 3 ). Table 1 shows the input data used along with their sources. 

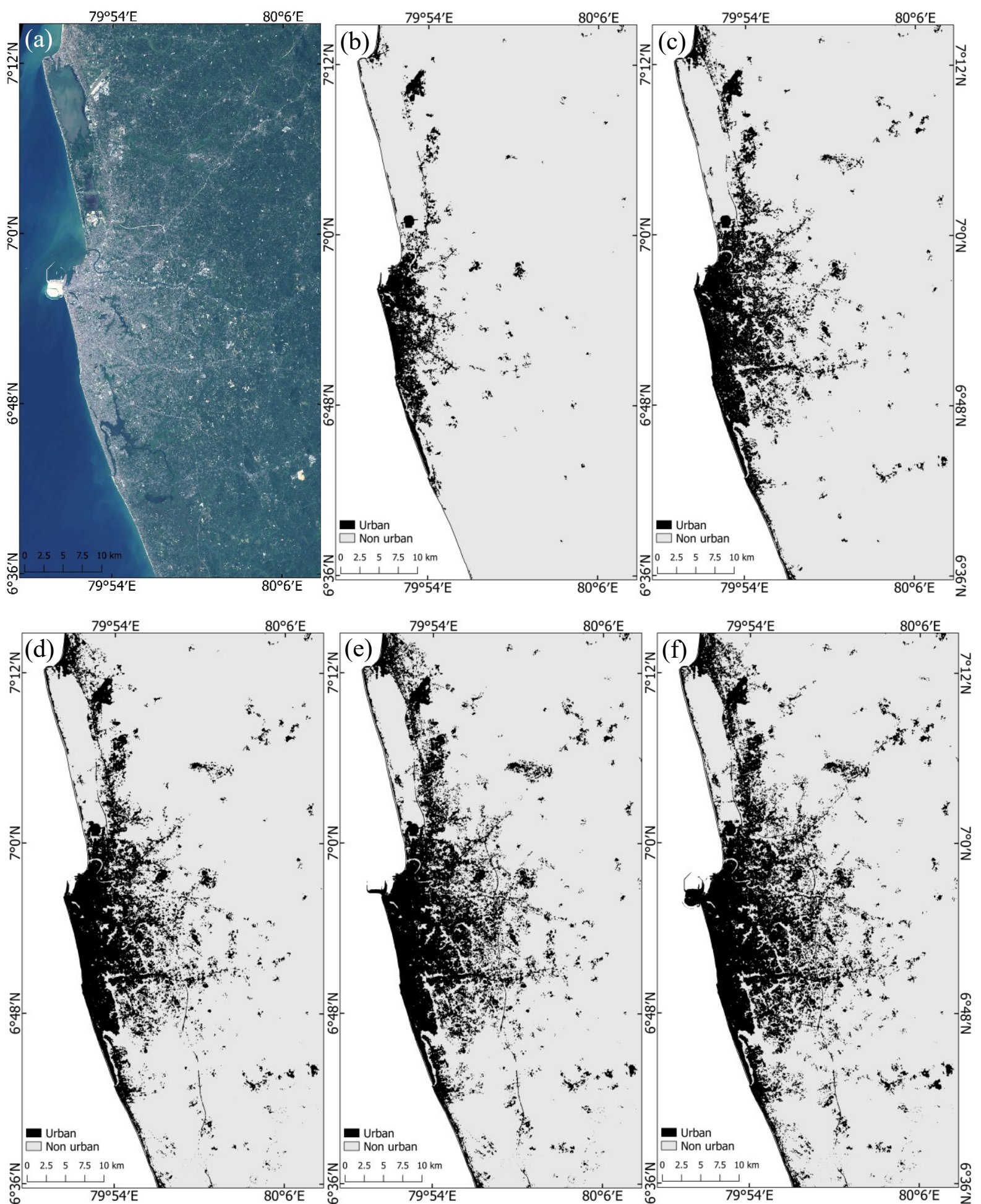

Figure 2: Input maps (a) True color Landsat OLI image in 2019, Urban area maps in (b) 1997, (c) 2005, (d) 2008, (e) 2014, (f) 2019 

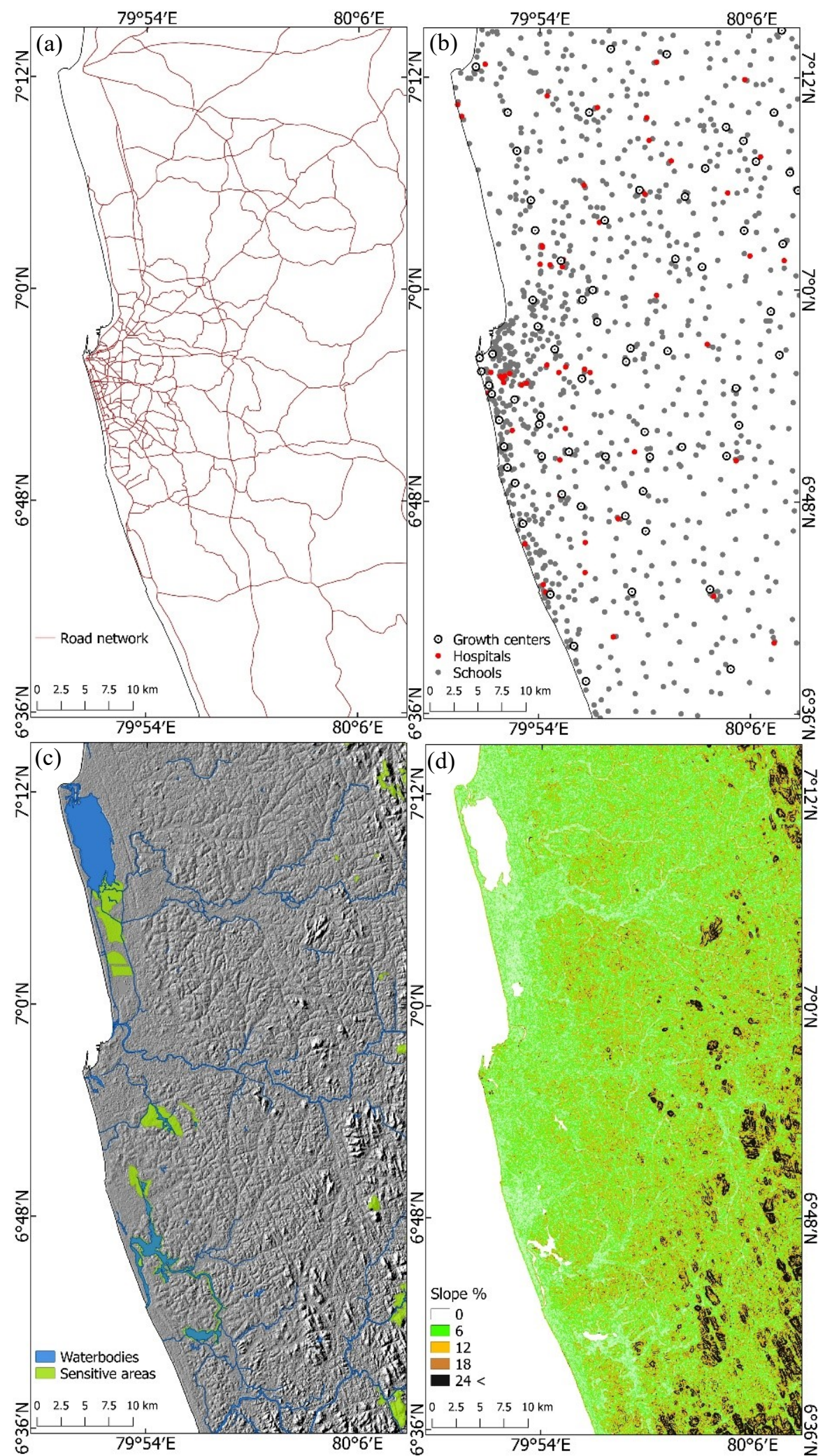

Figure 3: Predictor variables (a) Road network (b) Growth centers, Hospitals, Schools (c) Environmentally sensitive areas (d) Slope 
2.3. Urban Growth Models Used

(i) FUTURES: FUTURES model (Figure 4) (Meentemeyer et al., 2013) is a multilevel modelling framework consisting of three sub-models namely, POTENTIAL, DEMAND and Patch-Growing Algorithm (PGA). POTENTIAL sub-model quantifies the site suitability based on hypothesized environmental, infrastructural, and socioeconomic factors. DEMAND sub-model quantifies per capita urban land demand among sub-regions based on increase in population and concurrent urban change specific to each sub-region. PGA is a stochastic patch-growing algorithm that determines the shape, size and distribution of urban patches. Application of each sub-model was performed using r.futures extension in GRASS GIS (GRASS Development Team, 2020).

(ii) SLEUTH: SLEUTH model (Clarke et al., 1997) is a Cellular Automata (CA) based urban growth simulation. The name of the model is an acronym for the six layers of raster maps used as input data in the model namely, Slope, Landuse, Excluded, Urban, Transport, Hillshade. SLEUTH model uses these six layers as input to compute transitions over a given time period. The SLEUTH model assigns the state of urban or non-urban to each cell for urban growth simulation using four transition rules namely, spontaneous growth, new spreading center growth, edge growth, and road-influenced growth. These four urban growth rules work sequentially in each growth cycle and are controlled by five-growth coefficients, namely, diffusion, breed, spread, road gravity, and slope resistance. These growth coefficients need to be determined by calibration using at least four historical urban growth maps. The studies around the world have used either one metric or a weighted sum or product of several metrics to narrow down on suitable growth coefficients (Ilyassova et al., 2019). The SLEUTH model produces 13 different metrics as a measure of fit between modelled growth to the observed historical growth (Dietzel and Clarke, 2007). The schematic diagram of SLEUTH model and its components are shown in Figure 5.

(iii) MOLUSCE: MOLUSCE (NextGIS, 2012) is a CA based Open Source model developed as a plugin for QGIS. MOLUSCE stands for Modules for Land Use Change Simulations. It is a user-friendly QGIS plugin that affords users to perform urban growth modelling and simulations. Firstly, the model calculates the area of change based on the historical urban area maps supplied. The second step involves Transition Potential Modelling, where MOLUSCE provides choice among Artificial Neural Network, Multi-criteria Evolution, Weight of Evidence and Logistic Regression to derive transition potential. The CA algorithm simulate the urban growth based on area of change and transition potentials derived in first and second step.

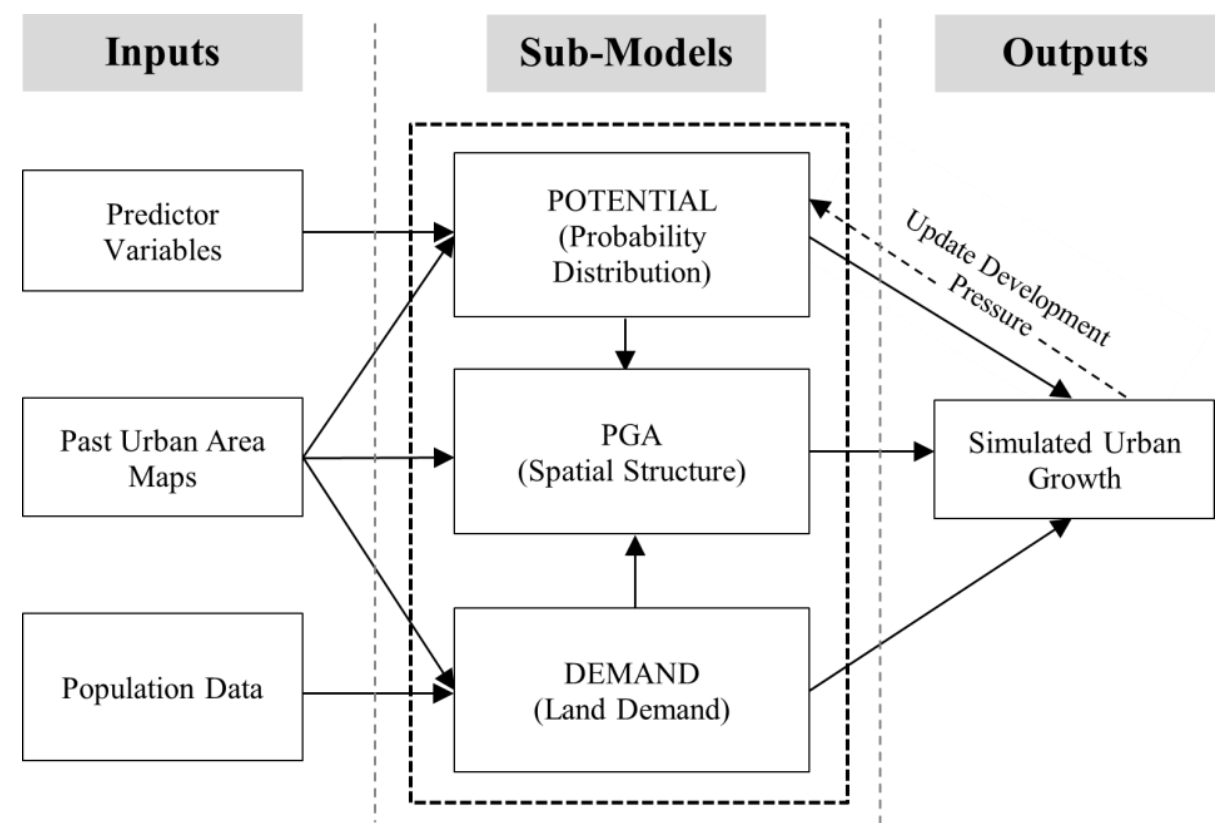

Figure 4: FUTURES modelling framework (after Meentemeyer et al., 2013) 


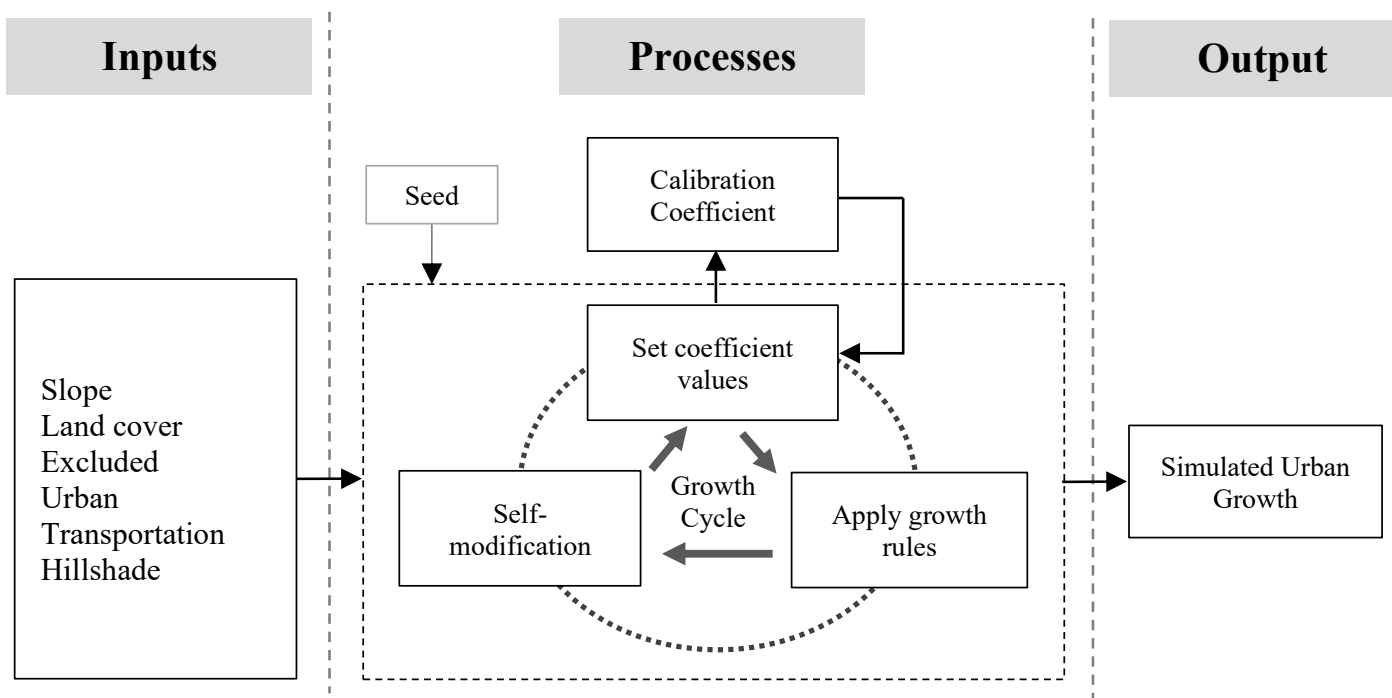

Figure 5: SLEUTH modelling framework (after Chaudhuri and Clarke, 2013)

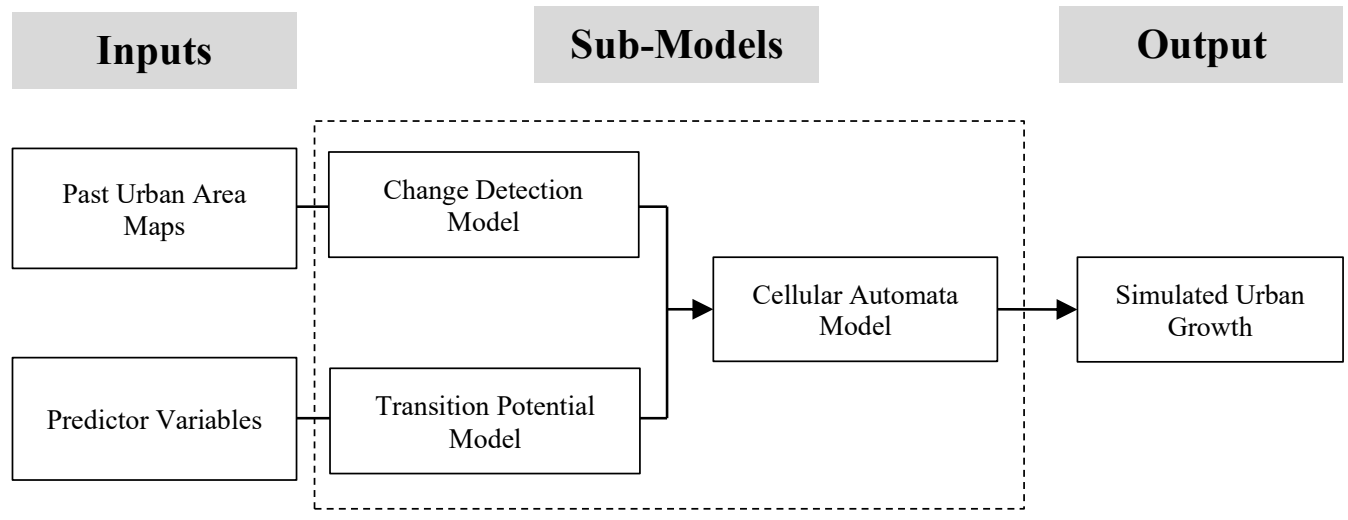

Figure 6: MOLUSCE modelling framework

MOLUSCE can only simulate the growth for a similar time iteration as historical urban maps provided to detect the urban change in the first step. In other words, if the user provides historic urban area maps with 10 -year gap, the model can simulate only for subsequent 10-year iterations of growth. MOLUSCE urban growth modelling steps are depicted in Figure 6.

\subsection{Model Implementation}

In this study, the three model namely FUTURES, SLEUTH and MOLUSCE were implemented on study area Colombo, using their simplest state i.e., minimum inputs and default settings to run the models.

(i) FUTURES: FUTURES model has integrated in GRASS GIS under ' $r$.futures' addon (Petrasova et al., 2016). In this study, r.futures.demand sub-model was used to estimate per-capita land demand of each subregion. The land area that would potentially be transformed into urban was estimated using population projection from 2008 to 2019. The projection for 2019 assumes status quo situation based on the extrapolated relationship between observed urban change and population during 1997, 2005 and 2008. The POTENTIAL sub-model runs under r.futures.potential module generates the transition potential surface describing the candidate locations for future development using Multilevel Logistic Regression (MLR). Transition potential surface is determined based on selected site suitability factors, distance to roads, distance to growth centers, distance to schools, distance to hospitals, distance to environmentally sensitive areas and percentage of slope (Figure 2). Based on identified site suitability and demand for new urban development, PGA determines the patch size and distribution for new patches. r.futures.pga is the module used for implementing the PGA. FUTURES model result of predicted urban map for 2019 using urban 2008 as base is shown in Figure 7(a). 


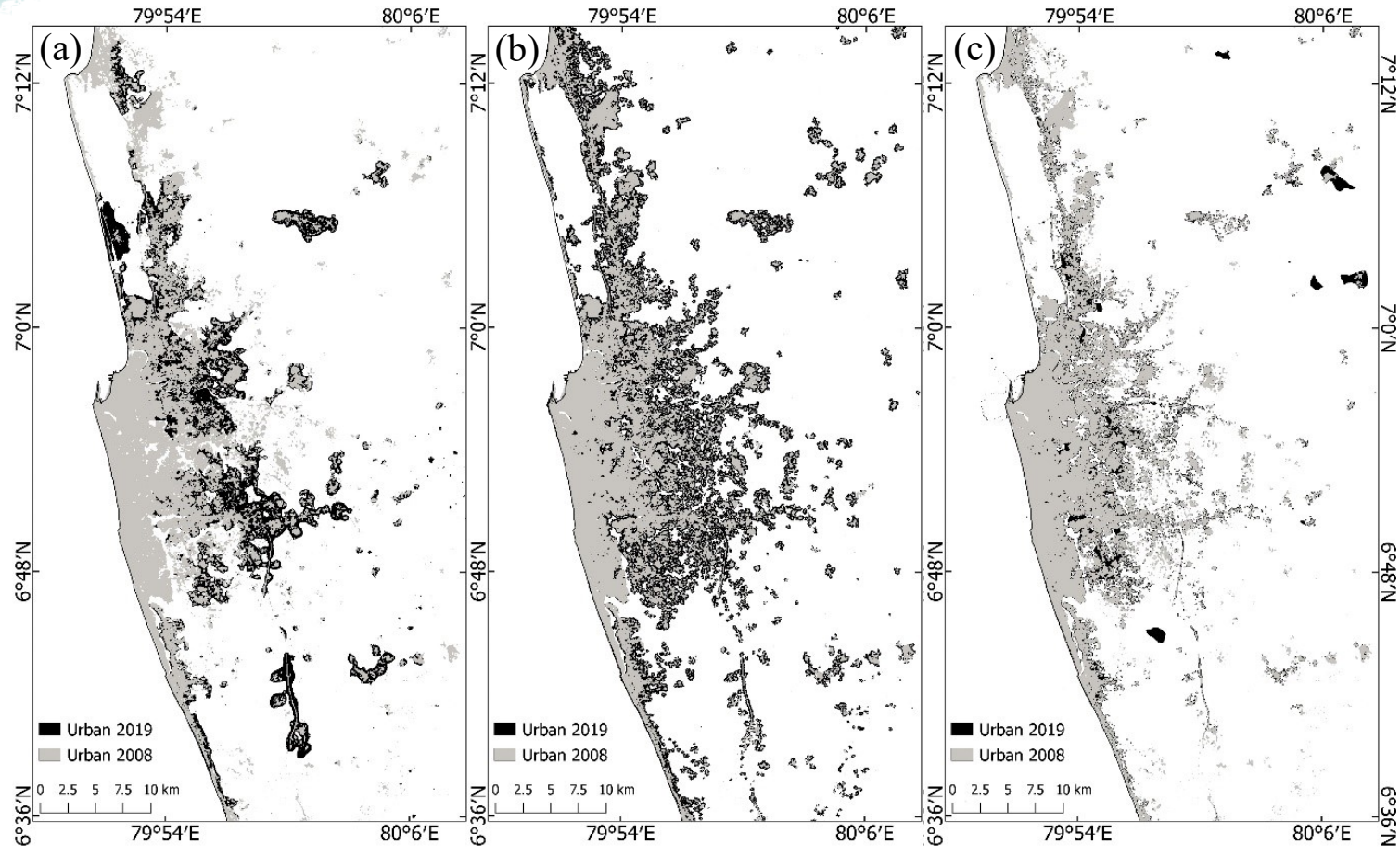

Figure 7: Simulated urban areas for 2019 by (a) FUTURES, (b) SLEUTH, (c) MOLUSCE

(ii) SLEUTH: SLEUTH model requires a minimum of four historic urban area maps, two maps of transportation network representing early and later stages, slope, hill shade and exclusion layer. The model is executed in three phases, namely, test phase used to verify the model; calibration phase used to derive a set of growth coefficients specific to an urban area under investigation; and prediction phase used to simulate the future urban growth of the study area. In the present case, the urban area maps for the year 1997, 2005, 2014 and 2019 along with the transportation network maps for the year of 1997 and 2019 were used. Further, the percentage of slope, an exclusion layer (exclusion of the water bodies) and a hill shade layer were provided as other inputs. In this study, brute force calibration along with the Population metric (least squares regression score for modeled urbanization compared to actual urbanization for the control years used in calibration) were used to estimate the calibration coefficients and evaluate the goodness of fit respectively. We have used an independent urban area map of 2008, which was not considered in calibration process of SLEUTH to initiate the prediction mode to simulate the urban growth from 2008 to 2019. The predicted urban area map of 2019 from SLEUTH model using urban 2008 as base is shown in Figure 7(b). (iii) MOLUSCE model: The MOLUSCE model has graphic user interface and FUTURES and SLEUTH models are command-line based. Input data for MOLUSCE model include urban area maps for 1997 and 2008 to estimate areas that have changed to urban during this time interval. Predictor variables used to include distance to roads, distance to growth centers, distance to schools, distance to hospitals, distance to environmentally sensitive areas and slope. The transition potential was estimated based on the Artificial Neural Networks using the default settings consisting of 10 hidden layers and 1000 training samples. Final step was CA simulation to generate the predicted urban map of the study area. The predicted urban area map of 2019 from MOLUSCE model using urban 2008 as base is shown in Figure 7(c).

\subsection{Model Validation}

All three models were initialized for simulation using urban area map 2008 to predict the urban area map of 2019. The predicted urban growth from 2008 to 2019 from three models were compared pixel by pixel with remote sensing derived urban growth map from 2008 to 2019 to produce confusion matrices. Confusion matrix is composed of Hits $(H)$, Misses $(M)$, False Alarms $(F A)$ and Correct Rejections $(C R)$. 
Where $H$ refers to observed change simulated as change, $M$ refers to observed change simulated as persistence, $C R$ refers to observed persistence simulated as persistence and $F A$ refers to observed persistence simulated as change. Confusion matrix used to estimate various validation metrics namely, Producer Accuracy $(P A)$ (Equation 1), User Accuracy (UA) (Equation 2), Overall Accuracy $(O A)$ (Equation 3), Specificity $(S)$ (Equation 4), Mathews Correlation Coefficient (MCC) (Equation 5), Figure of Merit (FoM) (Equation 6).

$$
\begin{array}{cr}
P A=\frac{H}{H+M} & \text { Equation 1 } \\
U A=\frac{H}{H+F A} & \text { Equation 2 } \\
O A=\frac{H+C R}{H+M+F A+C R} & \text { Equation 3 } \\
S=\frac{C R}{C R+F A} & \text { Equation 4 } \\
M C C=\frac{(H \times C R)-(F A \times M)}{\sqrt{H+F A)(H+M)(C R+F A)(C R+M)}} & \\
& \\
F M=\frac{H}{H+M+F A} & \text { Equation 5 }
\end{array}
$$

Equation 6

Producer's accuracy (PA) is the proportion of correctly simulated urban pixels by the model to observed urban pixels in the reference data, User Accuracy (UA) is the proportion of correctly simulated urban pixels to total number of simulated urban pixels by the model and Overall Accuracy $(\mathrm{OA})$ is the proportion of both correctly simulated urban and non-urban pixels to total number of pixels in the study area (Olofsson et al., 2014). The Matthews Correlation Coefficient (MCC) is a measure to assess the balanced quality of a model when categories of a map are in significantly different sizes (Boughorbel et al., 2017). The Figure of Merit (FoM) is a measure to examine how the simulated change overlaps with the reference change (Pontius et al., 2008). Specificity is used as a measure true negative rate that measures the model ability to avoid simulation of non-urban pixels that are not suitable for urbanization (Kantakumar et al., 2019).

\section{Simulation Results and Discussion}

In order to evaluate of FUTURES, SLEUTH and MOLUSCE for performance, we compared the simulated urban growth from 2008 to 2019 after masking the initial urban area 2008 from simulated urban 2019 with the observed urban growth from 2008 to 2019 derived from Landsat images. Simulated urban growth maps are shown in Figure 7. Urban growth models are approximation of complex urban system. Therefore, the validation of simulations produced by an urban growth models are essential to determine capability of models to replicate urban growth with sufficient accuracy (Kantakumar et al., 2019). As a first step in validation, we compared the simulated urban growth to observed urban growth of the study area (127.37 $\mathrm{km}^{2}$ ) during the 2008-2019 period. The FUTURES, SLEUTH and MOLUSCE models simulated urban growths as $148.91 \mathrm{~km}^{2}, 250.56 \mathrm{~km}^{2}$ and $77.11 \mathrm{~km}^{2}$ respectively. Among simulation results, SLEUTH model showed an over estimation and MOLUSCE model showed an under estimation while FUTURE model matched closely with the observed urban growth. The FUTURES model considers sub-region wise urban change and population growth to determine per capita land demand which could be the reason for better estimation of urban growth. The over estimation of SLEUTH model simulations could be as a result of permitting unrestricted growth at all locations (except water bodies) without considering other criteria for site suitability. Another reason for the overestimation of SLEUTH urban growth prediction can be explained by SLEUTH's rigid modeling requirements of four time-steps to assess changes which cannot be calibrated beyond linear extrapolations of the amount of change. SLEUTH's requirement of four time-steps determines the time period for linear extrapolation, where the amount of newly developed cells are regressed. User defined estimates can account for such changes (nonlinear trends) which is possible with FUTURES as it is the only model that allows user defined quantity of change in this study.

In addition, to the overall comparison of areas of simulated and observed urban growth, the model performances were also evaluated by making a pixelby-pixel comparison to produce confusion matrices. The results of validation were presented in the form of hits, misses, false alarms, correct rejections are presented in Figure 8 and Table 2, and the validation metrics are listed in Table 3. The results show that, the OA of all three models are over $90 \%$, indicating the higher agreement of simulated pixels for both urban and non-urban. 

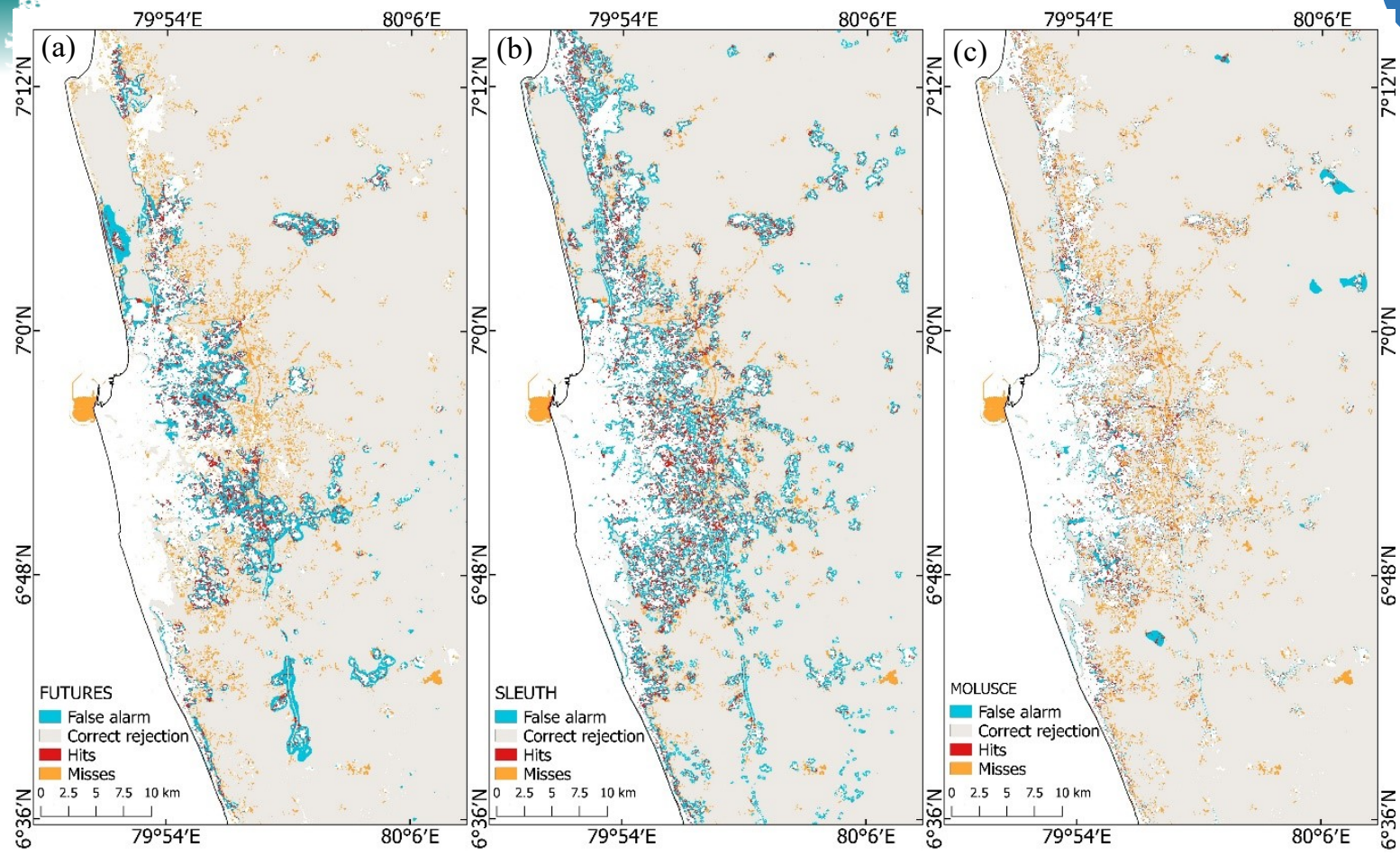

Figure 8: Validation maps for (a) FUTURES, (b) SLEUTH, and (c) MOLUSCE

Table 2: Elements of confusion matrix

\begin{tabular}{|l|r|r|r|r|r|r|}
\hline \multirow{2}{*}{} & \multicolumn{2}{|c|}{ FUTURES } & \multicolumn{2}{c|}{ SLEUTH } & \multicolumn{2}{c|}{ MOLUSCE } \\
\cline { 2 - 7 } & Area $\left(\mathrm{km}^{2}\right)$ & \multicolumn{1}{c|}{$\%$} & Area $\left(\mathrm{km}^{2}\right)$ & \multicolumn{1}{c|}{$\%$} & Area $\left(\mathrm{km}^{2}\right)$ & \multicolumn{1}{c|}{$\%$} \\
\hline Hit & 45.64 & 1.8 & 77.30 & 3.0 & 33.76 & 1.3 \\
\hline Misses & 81.74 & 3.2 & 50.08 & 2.0 & 93.62 & 3.7 \\
\hline False Alarm & 103.27 & 4.0 & 173.26 & 6.8 & 43.35 & 1.7 \\
\hline Correct Rejection & 2323.94 & 91.0 & 2253.95 & 88.2 & 2383.86 & 93.3 \\
\hline
\end{tabular}

Table 1: Validation metrics

\begin{tabular}{|l|c|c|c|c|c|c|c|}
\hline \multicolumn{1}{|c|}{ Models } & $\begin{array}{c}\text { Producer } \\
\text { Accuracy }\end{array}$ & $\begin{array}{c}\text { User } \\
\text { Accuracy }\end{array}$ & $\begin{array}{c}\text { Overall } \\
\text { Accuracy }\end{array}$ & Specificity & $\begin{array}{c}\text { Matthews } \\
\text { Correlation } \\
\text { Coefficient }\end{array}$ & $\begin{array}{c}\text { Figure of } \\
\text { Merit }\end{array}$ & Kappa \\
\hline FUTURES & 0.36 & 0.31 & 0.93 & 0.96 & 0.29 & 0.20 & 0.69 \\
\hline SLEUTH & 0.61 & 0.31 & 0.91 & 0.93 & 0.39 & 0.26 & 0.66 \\
\hline MOLUSCE & 0.27 & 0.44 & 0.95 & 0.98 & 0.31 & 0.20 & 0.75 \\
\hline
\end{tabular}

The OA of the MOLUSCE model is the highest compared to SLEUTH and FUTURES.It is important to note, the use of OA cannot be interpreted as a direct method of model efficacy, due to pervasiveness of non-urban area in the study area as compared to urbanized area (Kantakumar et al., 2019). Therefore, MCC was used to avoid the unbalanced effect of pervasiveness and change. The MCC is higher for SLEUTH (0.39) compared to FUTURES (0.29) and MOLUSCE (0.31). The PA and FoM values of SLEUTH is comparatively higher than other models, thereby indicating that SLEUTH can effectively simulate urban pixels with better spatial accuracy as compared with FUTURES and MOLUSCE. Using 2014 urban map in calibration stage of SLEUTH, which was not used in case FUTURES and MOLUSCE models, could be a reason behind predicting urban pixels with better spatial accuracy by SLEUTH. The over estimation of urban area by SLEUTH model could be reflecting in the higher MCC, FoM and PA values. The comparatively higher $\mathrm{OA}$ and $\mathrm{UA}$ reflected in MOLUSCE can be ascribed to the under estimation of urban growth in the model. However, previous study of FUTURES model implementation in Colombo (Jayasinghe et al., 2021) has shown a better 
overall accuracy with $30 \%$ overall disagreement (sum of quantity and allocation disagreement) and 0.73 kappa value. Moreover, that study accurately predicted the expected landscape expansion pattern type (infilling) within coming decade according to the diffusion and coalescence theory (Dietzel et al., 2005).

Apart from model performances, userfriendliness of FUTURES and MOLUSCE offers an advantage as these models are tightly integrated into Open Source GRASS and QGIS desktop GIS environment. SLEUTH model is Open Source standalone implementation and is not seamlessly integrated in desktop GIS environment. Such integration affords easier analysis and visualization of obtained results. SLEUTH model requires minimum number inputs and more computational resources for calibration in comparison to the FUTURES and MOLUSCE. Thus, taking all these trade-offs into account, it is difficult to unequivocally conclude either models as offering the best solution, especially when they have implemented with minimum required datasets and default settings.

\section{Conclusion}

Based on our study, we found that the implementation of MOLUSCE is easy in comparison to the FUTURES and SLEUTH. FUTURES is a robust, easily customizable model with flexibility in incorporation of complex policy scenarios through trends related to population dynamics and a range power functions to control transition potentials. The FUTURES model urban area estimates are closer to the observed changes as compared to SLEUTH and MOLUSCE. FUTURES offers the advantage of incorporating potential surface as well as per capita land demand via DEMAND sub-model which can be considered as the main reasons for its better performance in estimating urban growth. Although the SLEUTH calibration is a computationally intensive, we have found that the model is scalable and yield higher spatial accuracy in simulation. Incorporation of exclusion factor for potential surface generation could mitigate the overestimation of urban areas observed in the results of the SLEUTH model. Besides discussed comparison criteria, FUTURES and SLEUTH both models allow to test various urban growth scenarios such as status quo, infill growth and sprawl which is not available with MOLUSCE model.

The aim of the study was to evaluate the performance of FUTURES, SLEUTH and MOLUSCE models in their simplest case. The present results reveal that keeping the variations of implementation techniques and procedures involved in these models, it could be inappropriate to unequivocally conclude which model performed better. However, considering the tradeoff between computational overheads and obtained results, it could be suggested that FUTURES offers a good solution for simulation of urban growth. Our future studies will focus on customizing the models by using the same method for estimating the transition potential surface and incorporating into FUTURES, SLEUTH and MOLUSCE as exclusion layer in order to make more objective evaluation.

\section{References}

Boughorbel, S., Jarray. F. and El-Anbari, M., 2017, Optimal Classifier for Imbalanced Data Using Matthews Correlation Coefficient Metric. PLoS ONE, Vol. 12, No.6, 1-17.

Brenning, A., 2012, Spatial Cross-Validation and Bootstrap for the Assessment of Prediction Rules in Remote Sensing: The R package Sperrorest. In: 2012 IEEE International Geoscience and Remote Sensing Symposium, July 2012, 5372-5375.

Chaudhuri, G. and Clarke, K., 2013, The SLEUTH Land Use Change Model: A Review. Environmental Resources Research, Vol. 1, 88105.

Chaudhuri, G. and Clarke, K., 2019, Modeling an Indian Megalopolis-A Case Study on Adapting SLEUTH Urban Growth Model. Computers, Environment and Urban Systems, Vol. 77, 101358-101358.

Clarke, C., Hoppen, S. and Gaydos, L., 1997, A SelfModifying Cellular Automaton Model of Historical Urbanization in the San Francisco Bay Area. Environment and Planning B: Planning and Design, Vol. 24, No. 2, 247-261.

Dorning, M., Koch, J., Shoemaker, D. and Meentemeyer, R., 2015, Simulating urbanization scenarios reveals tradeoffs between conservation planning strategies. Landscape and Urban Planning, Vol. 136, 28-39.

Dietzel, C. and Clarke, C., 2007, Toward Optimal Calibration of the SLEUTH Land Use Change Model. Transactions in GIS, Vol. 11, No. 1, 2945.

Dietzel, C., Oguz, H., Hemphill, J., Clarke, K. and Gazulis, N., 2005, Diffusion and Coalescence of the Houston Metropolitan Area: Evidence Supporting a New Urban Theory. Environment and Planning B: Planning and Design, Vol. 32, No. 2, 231-246.

Gounaridis, D., Chorianopoulos, I. and Koukoulas, S., 2018, Exploring Prospective Urban Growth Trends under Different Economic Outlooks and Land-Use Planning Scenarios: The Case of Athens. Applied Geography, Vol. 90, 34-144. 
GRASS Development Team, 2020, Geographic Resources Analysis Support System (GRASS) Software, Version 7.2. Open Source Geospatial Foundation. Electronic document: http://grass.osgeo.org.

Hakim, A., Baja, S., Rampisela, D. and Arif, S., 2019, Spatial Dynamic Prediction of Landuse/ Landcover Change (Case Study: Tamalanrea Sub-District, Makassar City). IOP Conference Series: Earth and Environmental Science, Vol. 280, 1-9.

Ilyassova, A., Kantakumar, L. N. and Boyd, D., 2019, Urban Growth Analysis and Simulations Using Cellular Automata and Geo-Informatics: Comparison Between Almaty and Astana in Kazakhstan. Geocarto International, Vol. 36, No. 5, 520-539.

Jayasinghe, P., Raghavan, V. and Yonezawa, G., 2021, Exploration of Expansion Patterns and Prediction of Urban Growth for Colombo City, Sri Lanka. Spatial Information Research. 1-14.

Jin, J. and Lee, H-Y., 2018, Understanding Residential Location Choices: An Application of the UrbanSim Residential Location Model on Suwon, Korea. International Journal of Urban Sciences, Vol. 22, No. 2, 216-235.

Kantakumar, L., Kumar, S. and Schneider, K., 2019, SUSM: A Scenario-based Urban Growth Simulation Model Using Remote Sensing Data. European Journal of Remote Sensing. Vol. 52, No. 2, 26-41.

Kantakumar, L. N., Sawant, N. G. and Kumar, S., 2011, Forecasting Urban Growth Based on GIS, RS and SLEUTH Model in Pune Metropolitan Area. International Journal of Geomatics and Geosciences, Vol. 2, No. 2, 568-579.

Meentemeyer, R. K., Tang, W., Dorning, M. A., Vogler, J. B., Cunniffe, N. J. and Shoemaker, D. A., 2013, FUTURES: Multilevel Simulations of Emerging Urban-Rural Landscape Structure Using a Stochastic Patch-Growing Algorithm. Annals of the Association of American Geographers, Vol.103, No. 4, 785-807.

NextGIS, 2012, MOLUSCE: QGIS Python Plugins Repository. Available at: https://plugins.qgis.org/plugins/molusce/ (accessed 9 November 2020).

Nugroho, A. B., Hasyim, A. W. and Usman, F., 2018, Urban Growth Modelling of Malang City using Artificial Neural Network Based on MultiTemporal Remote Sensing. Civil and Environmental Science Journal, Vol. I, No. 2, 52 -61 .

Olofsson, P., Foody, G. M., Herold, M., Stehman, S.V., Woodcock, C. E. and Wulder, M. A., 2014, Good Practices for Estimating Area and
Assessing Accuracy of Land Change. Remote Sensing of Environment, Vol. 148, 42-57.

Petrasova, A., Petras, V., Van Berkel, D., Harmon, B. A., Mitasova, H. and Meentemeyer R., 2016, Open Source Approach to Urban Growth Simulation. The International Archives of the Photogrammetry, Remote Sensing and Spatial Information Sciences, 953-959.

Pickard, B., Gray, J. and Meentemeyer, R., 2017, Comparing Quantity, Allocation and Configuration Accuracy of Multiple Land Change Models. Land, Vol. 6, No. 3(52), 1-21.

Pontius, R., Boersma, W., Castella, J., Clarke, K., de Nijs, T., \& Dietzel, C., Duan, Z., Fotsing, E., Goldstein, N., Kok, K., Koomen, E., Lippitt, C. D., Mcconnell, W., Mohd Sood, A., Pijanowski, B., Pithadia, S., Sweeney, S., Trung, T. N., Veldkamp, A. T. and Verburg, P. H., 2008, Comparing the input, output, and validation maps for several models of land change. The Annals of Regional Science, Vol. 42, No.1, 11-37.

Pontius, G. R. and Malanson, J., 2005, Comparison of the Structure and Accuracy of Two Land Change Models. International Journal of Geographical Information Science, Vol. 19, No. 2, 243-265.

Pontius, R. G. and Millones, M., 2011, Death to Kappa: Birth of Quantity Disagreement and Allocation Disagreement for Accuracy Assessment. International Journal of Remote Sensing, Vol. 32, No.15, 4407-4429.

Sandamali, S. P. I., Kantakumar, L. N. and Sivanantharajah, S., 2018, Remote Sensing Data and SLEUTH Urban Growth Model: As Decision Support Tools for Urban Planning. Chinese Geographical Science, Vol. 28, No. 2, 274-286.

Shoemaker, D., BenDor, T. and Meentemeyer, R. 2019, Anticipating Trade-offs Between Urban Patterns and Ecosystem Service Production: Scenario Analyses of Sprawl Alternatives for a Rapidly Urbanizing Region. Computers, Environment and Urban Systems, Vol. 74, 114125.

Saxena, A. and Jat, M. K., 2020, Analysing Performance of SLEUTH Model Calibration Using Brute Force and Genetic Algorithm-Based Methods. Geocarto International, Vol. 35, No. 3, 256-279.

Van Berkel, D., Shashidharan, A., Mordecai, R., Vatsavai, R., Petrasova, A., Petras, V., Mitasova, H., Vogler, J. B. and Meentemeyer, R., 2019, Projecting Urbanization and Landscape Change at Large Scale Using the FUTURES Model. Land, Vol. 8, No. 10, 1-28. 